\title{
Acetylcholine Elevation Relieves Cognitive Rigidity and Social Deficiency in a Mouse Model of Autism
}

\author{
Golan Karvat' and Tali Kimchi*,' \\ 'Department of Neurobiology, Weizmann Institute of Science, Rehovot, Israel
}

\begin{abstract}
Autism spectrum disorders (ASD) are defined by behavioral deficits in social interaction and communication, repetitive stereotyped behaviors, and restricted interests/cognitive rigidity. Recent studies in humans and animal-models suggest that dysfunction of the cholinergic system may underlie autism-related behavioral symptoms. Here we tested the hypothesis that augmentation of acetylcholine (ACh) in the synaptic cleft by inhibiting acetylcholinesterase may ameliorate autistic phenotypes. We first administered the acetylcholinesterase inhibitor (AChEl) Donepezil systemically by intraperitoneal (i.p.) injections. Second, the drug was injected directly into the rodent homolog of the caudate nucleus, the dorsomedial striatum (DMS), of the inbred mouse strain BTBR $T+$ tf/J (BTBR), a commonly-used model presenting all core autism-related phenotypes and expressing low brain ACh levels. We found that i.p. injection of AChEl to BTBR mice significantly relieved autism-relevant phenotypes, including decreasing cognitive rigidity, improving social preference, and enhancing social interaction, in a dose-dependent manner. Microinjection of the drug directly into the DMS, but not into the ventromedial striatum, led to significant amelioration of the cognitive-rigidity and social-deficiency phenotypes. Taken together, these findings provide evidence of the key role of the cholinergic system and the DMS in the etiology of ASD, and suggest that elevated cognitive flexibility may result in enhanced social attention. The potential therapeutic effect of AChEls in ASD patients is discussed. Neuropsychopharmacology (2014) 39, 831-840; doi: I0.1038/npp.2013.274; published online 30 October 2013
\end{abstract}

Keywords: Autism; acetylcholine; dorsomedial striatum; mouse model; donepezil; BTBR

\section{INTRODUCTION}

Autism spectrum disorders (ASD) are diagnosed by behavioral deficiencies in social interactions, communication impairments, repetitive behaviors, and cognitive rigidity (Association AP, DSM-IV, 2000). The disorders have a strong genetic basis (Hallmayer et al, 2011), yet the underlying genetic and neuronal alternations are not well understood. Consequently, approved medications for treatment of ASD, eg, risperidone, atomoxetine, and selective serotonin reuptake inhibitors, address only associated, but not core, symptoms of the disorder (reviewed in (Canitano and Scandurra, 2011)), and no consistent biomarkers have been identified.

The cholinergic system is one such potential constituent. In the central nervous system (CNS), acetylcholine (ACh) is essential in evaluating the rewarding or threatening valence of stimuli and changes in environment, and responding accordingly (Pepeu and Giovannini, 2004). Therefore, it has a role in regulating behaviors relevant to ASD including attention (Arnold et al, 2002), cognitive flexibility

\footnotetext{
*Correspondence: Dr T Kimchi, Department of Neurobiology, Weizmann Institute of Science, 234 Herzel Street, Rehovot 76100, Israel, Tel: +972 893462 16, Fax: +972 89346357 ,

E-mail: tali.kimchi@weizmann.ac.il

Received 31 May 2013; revised 30 September 2013; accepted I October 2013; accepted article preview online 7 October 2013
}

(Ragozzino et al, 1998), social interactions (Avale et al, 2011), and stereotypical behaviors (McConville et al, 1992).

A wealth of evidence associates the cholinergic system with ASD. For example, neurons in a basal forebrain cholinergic nucleus of ASD patients are unusual in size, number, and structure (Kemper and Bauman, 1998). In addition, decreased concentrations of choline, a precursor of ACh and nicotinic-cholinergic receptor (nAChR) agonist, were found in ASD patients (Friedman, 2006), and low levels of cytosolic choline were correlated with the severity of autism (Sokol et al, 2002). Postmortem immunohistochemical studies revealed reduction of several nAChR subunits and M1 type muscarinic-cholinergic receptors (mAChR) in the neocortex, cerebellum, thalamus, and striatum of ASD patients (Deutsch et al, 2010; MukaetovaLadinska et al, 2010). nAChR abnormalities were correlated with ASD also in the genetic level, as CHRNA7, the gene encoding the $\alpha 7 \mathrm{nAChR}$ subunit, was found mutated in one ASD patient (Mikhail et al, 2011) and duplicated in 13 others (Leblond et al, 2012). Furthermore, deletion of the gene Chrnb2 encoding the $\beta 2$-subunit of nAChR (Granon et al, 2003), M1 type mAChR inhibition (McCool et al, 2008), and lesion of cholinergic cells (Walker et al, 2007) leads to autism-related behaviors in rodents.

One method to target the cholinergic system is to modulate its receptors, and preliminary studies focusing nicotinic receptors suggest improvement in the cognitive functions in ASD, yet it is not clear whether agonists or antagonists should be used (Mukaetova-Ladinska et al, 
2010). A more common and validated method to increase $\mathrm{ACh}$ availability in the brain is inhibiting acetylcholinesterase (AChE), and numerous studies revealed improvement in the cognitive abilities of attention, memory, and reversal learning following treatment with AChE inhibitors (acetylcholinesterase inhibitor, AChEI, eg, Donepezil, reviewed in (Yoo et al, 2007)). Most studies focused on the impact on Alzheimer's disease, yet a handful of studies examined the effect of the CNS-specific AChEI Donepezil on ASD symptoms. In two open-label (Buckley et al, 2011; Hardan and Handen, 2002) and one case-study (Srivastava et al, 2011), the drug was found useful in reducing overall ASD severity. Two double-blind placebo-controlled studies were conducted (Chez et al, 2003; Handen et al, 2011), showing amelioration in ASD symptoms after treatment with the drug. However, between-groups data were not analyzed (Chez et al, 2003) or were not statistically significant (Handen et al, 2011). Hence, the potential therapeutic effects of Donepezil on ASD symptoms in human patients are suggestive yet not conclusive (Handen et al, 2011).

Surprisingly, a systematic examination on the effects of AChEIs on autism-related symptoms in animal models was never done. Therefore, in the current study we set to examine the effect of AChEI on three core autistic-like behaviors in mice. As an animal model, we used the BTBR $\mathrm{T}+\mathrm{tf} / \mathrm{J}$ mouse strain, which is considered a reliable preclinical model for autism due to its distinct behavioral abnormalities resembling ASD symptoms including impaired social behavior, aberrant communication, increased repetitive behavior, and increased cognitive rigidity (reviewed in (Chadman and Guariglia, 2012)). Importantly, recent studies identified abnormally lower basal levels of brain ACh in BTBR mice compared with C57BL/6 mice (McTighe et al, 2013).

In the current study, we first sought to test the therapeutic potential of administrating Donepezil systemically on autism-related behaviors. Second, ACh activity in the caudate nucleus is essential for flexible planning and execution of goal-directed behaviors, according to environmental cues (Grahn et al, 2008), and abnormalities of this region were found in ASD (Sears et al, 1999). Hence, we also tested the effect of administration of the drug directly to the rodent homolog of the caudate nucleus, the dorso-medial striatum (DMS). We measured the behavioral outcome of the systemic and site-specific administrations in a set of tests aimed to assess cognitive rigidity, social deficiency, and repetitive behavior, as previously reported (Karvat and Kimchi, 2012).

\section{MATERIALS AND METHODS}

\section{Subjects}

Male BTBR $\mathrm{T}+\mathrm{tf} / \mathrm{J}$ (BTBR, Jackson Laboratory) and FVB/ NJ (FVB, Harlan Laboratories) were housed in groups of 3-5 littermates per cage, in humidity- and temperaturecontrolled room with reversed $12 \mathrm{~h}$ light cycle. Drug administration and surgical procedures started at 7 weeks of age and behavior testing at 8 weeks of age. All experimental procedures were approved by the Institutional Animal Care and Use Committees of the Weizmann Institute of Science.

\section{Drug Treatments}

Donepezil hydrochloride monohydrate (Sigma-Aldrich) was applied by either systemic intraperitoneal (i.p.) injection (experiment 1 ) or stereotactic intracranial injection into the DMS or the ventromedial striatum (VMS) (experiment 2).

\section{Experiment 1: Systemic I.P Injection}

For experiment 1 , the drug was dissolved in saline $(\mathrm{NaCl}$ $0.9 \%$ ). Mice received daily i.p. injections of $4 \mathrm{ml} / \mathrm{kg} 7$ days before and throughout the whole experiment $30 \mathrm{~min}$ before testing. Mice were randomly assigned to three treatment groups of littermates ( $N=8 /$ dose): 0.0 (saline, control), 0.3 , and $1.0 \mathrm{mg} / \mathrm{kg}$. During the time between drug administration and testing, the mice were held in the testing room. This sub-chronic administration regimen was chosen due to three reasons: (a) future treatment in humans is expected to be chronic, (b) previous studies with the drug (eg, (Van Dam et al, 2005)) utilized similar regimens, and (c) due to the continuation of the behavioral testing over a few days, an acute treatment was not possible. Furthermore, an additional group $(N=8)$ received $1.0 \mathrm{mg} / \mathrm{kg}$ (i.p.) of the drug in a semi-acute fashion, similar to the drug treatment in experiment 2 (see below).

\section{Experiment 2: Stereotactic Intracranial Injection}

Cannula implantation. Mice were anesthetized with $6 \mathrm{ml} /$ $\mathrm{kg}$ ketamine/xylazine solution, and secured to the stereotactic frame (Kopf). The coordinates of DMS were $1.0 \mathrm{~mm}$ anterior to bregma, $\pm 1.4 \mathrm{~mm}$ lateral to midline and $3.1 \mathrm{~mm}$ ventral to dura. Coordinates of the VMS were: anterior-posterior, $+1.0 \mathrm{~mm}$; medial-lateral, $\pm 1.4 \mathrm{~mm}$; and dorsal-ventral, $-4.5 \mathrm{~mm}$ (Franklin and Paxinos, 1997). Stainless-steel guide cannulae covered with dummy cups extending $1 \mathrm{~mm}$ ventrally were implanted at a $15^{\circ}$ angle aimed medially, and secured with screws covered with dental acrylic cement. Following the surgery, mice were singly housed and allowed to recover in their home-cages for 10 days, before behavioral testing commenced.

Drug treatment. Donepezil was dissolved in artificial cerebrospinal fluid (aCSF) (in mmol/l: $125 \mathrm{NaCl}$; $2.5 \mathrm{KCl}$; $\left.0.5 \mathrm{NaH}_{2} \mathrm{PO}_{4} ; 5 \mathrm{Na}_{2} \mathrm{HPO}_{4} ; 1 \mathrm{MgCl}_{2} ; 1.2 \mathrm{CaCl}, \mathrm{pH} 7.3\right)$. Seven mice were injected bilaterally with $100 \mathrm{ng}$ Donepezil in $0.5 \mu \mathrm{l}$ aCSF into the DMS. Two groups were used for control: mice that received $0.5 \mu \mathrm{laCSF}$ into the DMS $(N=6)$ and mice that received $100 \mathrm{ng}$ Donepezil in $0.5 \mu \mathrm{l}$ aCSF into the VMS $(N=7)$, as control for site specificity.

Drug microinjection. Each mouse received a bilateral injection $30 \mathrm{~min}$ before testing and stayed in the testing room until test onset for acclimation. The injection was carried out via internal cannula that extended $1.0 \mathrm{~mm}$ below the guide cannula. Polyethylene FEP tubing (CMA microdialysis) connected the cannulae to $50 \mu \mathrm{l}$ Hamilton syringes. The syringes were driven by a microinjection pump (Harvard Apparatus). Solutions were injected at a rate of $0.25 \mu \mathrm{l} / \mathrm{min}$ for $2 \mathrm{~min}$. The cannula remained in the guide cannula for $1 \mathrm{~min}$ after the injection to allow for diffusion. All mice were injected with aCSF in the third running day 
(see behavioral testing below) for acclimation to procedure and reduction of stress. In each day from the fourth running day until the end of experiment, mice were injected with either aCSF or Donepezil, according to group. A $30 \mathrm{~min}$ post-injection test time was chosen based on the results of a previously reported study (Wang and Tang, 1998).

Behavioral testing. The set of autism-related behavior tests was described previously in detail (Karvat and Kimchi, 2012) and consisted of the following: (I) running/jammed wheel series of tests; (II) open-field; (III) repetitive behavior measurement; (IV) male-male social interaction assay; (V) three-chamber sociability assay; and (VI) water T-maze. All experiments took place during the active period of the subjects in the dark (except for the T-maze, which was conducted under white fluorescent lighting) and were recorded by low-light sensitive video cameras under infrared illumination. Behaviors were scored by an experienced evaluator blind to treatment. The experimental procedures are given in the Supplementary Materials and Methods and are described below briefly.

Running/jammed wheel test. Mice were habituated to run on a wheel for 4 days (days Run 1-4). In the 5th and 6th days, the wheel was jammed (days Jam1-2). Comparison of interaction time with the wheel trying to move it in day Jam1 to running duration in day Run4 served as an indicator of ability to adjust to an environmental change. Comparison of day Jam2 to Jam1 served as indicator of memory of the change.

'Social' running wheel test. In the last day of the wheel assay, a stranger mouse was placed in the cage. Social preference was assessed by comparing interaction time with the stranger mouse, initiated by the subject mouse, compared with interaction time with the object (the jammed-wheel).

Open-field test. Subjects were put in the open-field cage for $30 \mathrm{~min}$. Total distance moved and time spent in the central compartment of the cage were measured.

Repetitive behaviors scoring. For repetitive self-grooming measure, subjects were put in a clean, empty cage. For repetitive digging measure, subjects were put in a similar cage covered with $\sim 1 \mathrm{~cm}$ corn-cob-bedding. Total repetitive behavior time was scored by an observer.

Male-male social interaction test. Alien ICR male mouse (5 weeks old) was introduced to a cage with the subject mouse. Behaviors scored included duration of sniffing, chasing, mounting, and aggressive attacks.

Three-chamber social approach and social novelty preference test. The test was conducted according to a previously described method (Moy et al, 2007). Following habituation, subject mouse was put in the central compartment of a three-chambered cage, and was allowed to interact with either a wire-cage containing a stranger mouse or an empty wire-cage. In the next trial, the subject had the choice between the already familiar mouse and a novel stranger. Duration of sniffing of each cage was scored.
Water T-maze spatial reversal learning test. The test was based on (Dong et al, 2005; Guariglia et al, 2011), and lasted 4 days, 10 trials per day. In the first 2 days, subjects had to learn and remember the arm containing the escape platform in a T-shaped water maze. In the third day, the arm containing the platform was changed. Numbers of correct turns and latency to reach the platform were measured.

\section{Histology: Confirmation of the Injection Site in the Brain}

Following the completion of behavioral testing, mice were euthanized and $0.5 \mu$ RhodamineB dye (Sigma-Aldrich) was delivered through each of the guide cannulae. The brains of the mice were removed and stored in a $4 \%$ formaldehyde solution at $4{ }^{\circ} \mathrm{C}$ for $72 \mathrm{~h}$. Using a vibratome (Leica), the brains were sliced into coronal sections $(100 \mu \mathrm{m})$ and photographed through a binocular. The sections were then examined to determine the placement of the cannula tips and assess the maximal spread.

\section{Statistical Analysis}

Data in figures are presented as mean \pm SEM. Comparisons between treatment groups were conducted using one-way or two-way repeated measure analysis of variation (ANOVA), with the Tukeys post hoc where appropriate. Statistical significance was set at $\alpha<0.05$ in the Statistica software (Statsoft, Tulsa, OK), when $p<0.05$ was considered significant.

\section{RESULTS}

\section{Experiment 1}

Systemic administration of Donepezil relieved cognitive rigidity in a dose-dependent manner. Mice injected i.p. with Donepezil $(0.3$ or $1.0 \mathrm{mg} / \mathrm{kg}$ ) showed a dose-dependent improvement in the ability to adjust to changes in the environment as evaluated by two independent behavioral measures: water T-maze, which tested reversal spatial learning (Figure 1a), and the running/jammed wheel assay, which tested the ability to adjust to a denial of a rewarding activity (Figure 1c).

In the water T-maze, all groups exhibited intact learning and memory capabilities, manifested in a significant improvement in correct turns taken between days 1 and 2 (Figure $1 b, F_{1,21}=48.8, p<0.001$ and $p<0.05$ for each group in post hoc analysis) with no difference between groups ( $p>0.7$ in each day). In addition, all groups exhibited similar durations to reach the platform in these days (Supplementary Figure S1, $p>0.5$ ). However, on day 3, when the arm containing the escape platform was changed, mice treated with Donepezil took significantly more correct turns $\left(\mathrm{F}_{2,21}=5.9, p<0.01\right)$, indicating improved adjustment to change. Post hoc analysis revealed a significant $(p<0.01)$ difference between mice treated with $1.0 \mathrm{mg} / \mathrm{kg}$ Donepezil compared with control saline-treated mice, and a smaller yet significant $(p<0.05)$ improvement in the group treated with $0.3 \mathrm{mg} / \mathrm{kg}$ (Figure $1 \mathrm{~b}$ ). Furthermore, analysis of preservative errors (ie, number of trials in which the previously reinforced arm is initially chosen until the first 


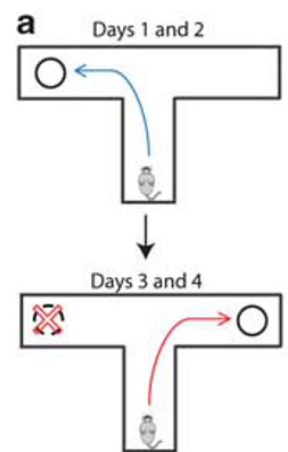

b
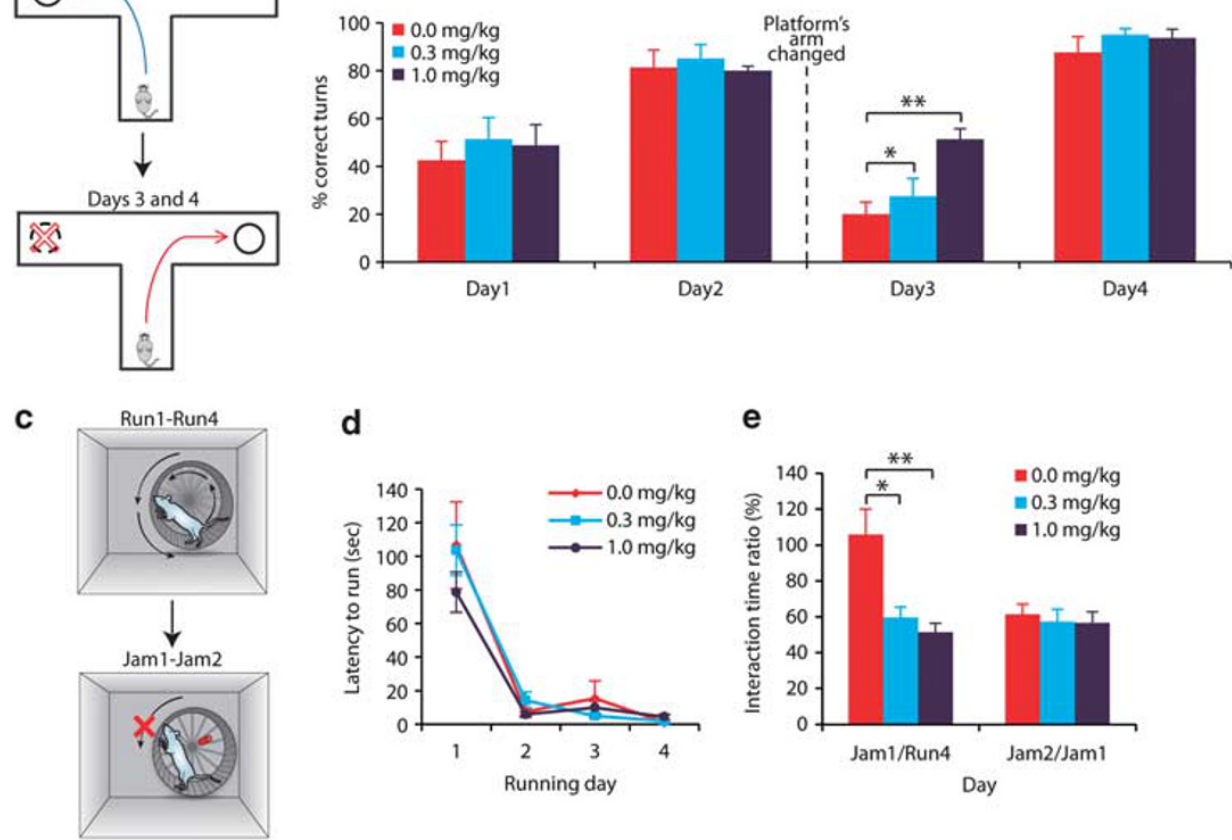

d

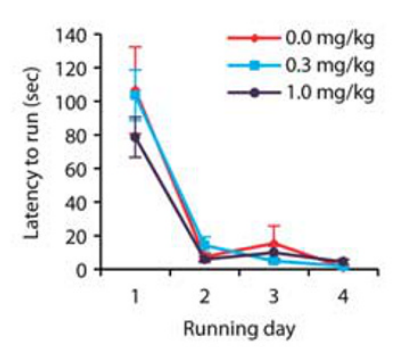

e

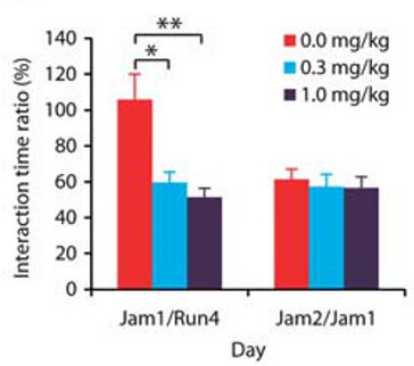

Figure I Intraperitoneal (i.p.) administration of Donepezil relieved cognitive rigidity in a dose-dependent manner. BTBR mice were injected i.p. with Donepezil $(0.3$ or $1.0 \mathrm{mg} / \mathrm{kg})$ or saline $(0.0 \mathrm{mg} / \mathrm{kg})$ for 7 days before testing and 30 min before each testing day. (a) Scheme of the water T-maze test. On the first two days, mice had to learn and remember the location of the arm containing an escape platform (upper panel). On day 3, the platform was moved to the opposite arm (lower panel), and the reversal learning was tested in 2 consecutive days. (b) Percentage of correct turns leading to the platform taken in each day. (c) Scheme of the running/jammed wheel assay. Mice had 4 consecutive days of access to a running wheel (Run I-Run4, upper panel), which was later jammed for 2 consecutive days (Jam I-Jam2, lower panel). (d). Acquisition of the running habit was assessed by the latency to start running in each of the running days. (e) The ratio between interaction times in days Jaml and Run4 (Jam I/Run4, left) indicates adjustment to the change. The ratio between interaction times in days Jam2 and Jaml (Jam2/Jaml, right) indicates memory of the change. Data are presented as mean \pm SEM. * $p<0.05$, ** $p<0.01$, $N=8 /$ dose

correct turn trial) showed a significant effect of treatment group $\left(\mathrm{F}_{2,21}=5.8, p<0.01\right)$. Number of preservative errors of mice treated with $1.0 \mathrm{mg} / \mathrm{kg}$ Donepezil $(2.75 \pm 0.63)$ was significantly lower $(p<0.01)$ than saline-treated mice $(6.38 \pm 0.58)$, and in mice treated with $0.3 \mathrm{mg} / \mathrm{kg}$ there was a trend $(p=0.069)$ toward lower number of preservative errors $(5.38 \pm 0.88)$. A significant effect for treatment groups was found also in latency to reach the platform (Supplementary Figure $\mathrm{S} 1, \mathrm{~F}_{2,21}=4.7, p<0.05$ ), when mice treated with $1.0 \mathrm{mg} / \mathrm{kg}$ Donepezil reached the platform faster $(p<0.05)$ compared with control group. Finally, mice from all treatment groups exhibited similar memory of the environmental change, manifested in improvement in correct turns taken between days 3 and $4\left(\mathrm{~F}_{1,21}=236\right.$, $p<0.001$ and $p<0.001$ for each group in post hoc analysis) with no difference between groups in day $4(p>0.4)$ as well as similar latency to reach the platform (Figure $1 \mathrm{~b}$ and Supplementary Figure S1, $p>0.4$ ).

In the running/jammed wheel assay, mice from all groups acquired the running habit as demonstrated by shorter latencies to start running on the wheel in days 2-4 compared with the first day (Figure $1 \mathrm{~d}, \mathrm{~F}_{3,63}=50.1$, $p<0.001$ for each group), with no differences between groups $(p>0.5)$. Saline-treated control mice, however, exhibited cognitive rigidity as they interacted with the wheel for similar amounts of time whether it was free or jammed (Figure 1e), in line with a previous report with untreated BTBR mice (Karvat and Kimchi, 2012). The ratio between wheel interaction times in the first jammed-day and last running day (Jam1/Run4), an indicator of the ability to adjust to an environmental change, was significantly lower in mice treated with Donepezil compared with control mice $\left(\mathrm{F}_{2,21}=8.6, p<0.01\right)$, in a dose-dependent manner, indicating improvement in cognitive flexibility in the Donepezil-treated groups. Mice from all treatment groups exhibited similar memory of the change, as indicated by the wheel interaction time ratio of Jam2/ Jam1 (Figure 1e, $p>0.8$ ).

Systemic administration of Donepezil improved sociability in a dose-dependent manner. The effect of i.p. administration of Donepezil on sociability was measured in three separate and complementary tests (Figures 2 and 3): the three-chamber test for social approach and novelty preference, the running wheel test for social interaction preference, and the male-male social interaction test.

In the three-chamber test, all groups spent similar amounts of time in each side-chamber during the habituation trial ( $p>0.9$ for each group). Number of entries to side chambers was similar as well $(p>0.5)$, supporting unchanged general activity. In the preference for social approach trial (Figure 2a), however, a significant interaction between stimulus (object or stranger) and Donepezil dosage was found (Figure $2 \mathrm{~b}, \mathrm{~F}_{2,21}=8.7, p<0.01$ ). While salinetreated mice showed no preference of the stranger $(p=1.0)$, 
a

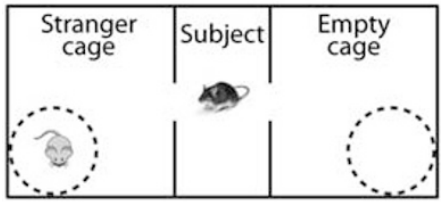

d

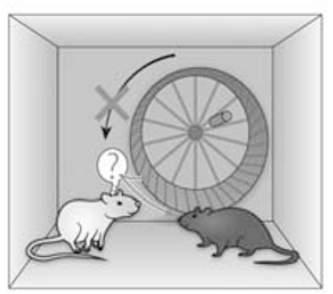

b
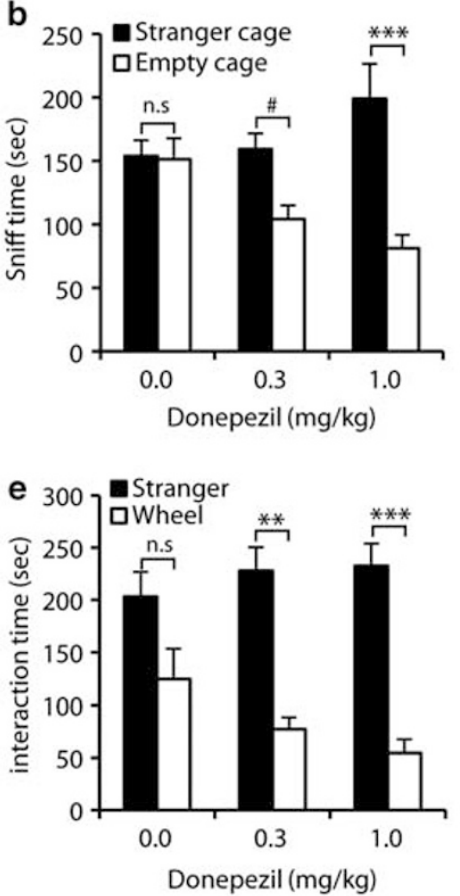
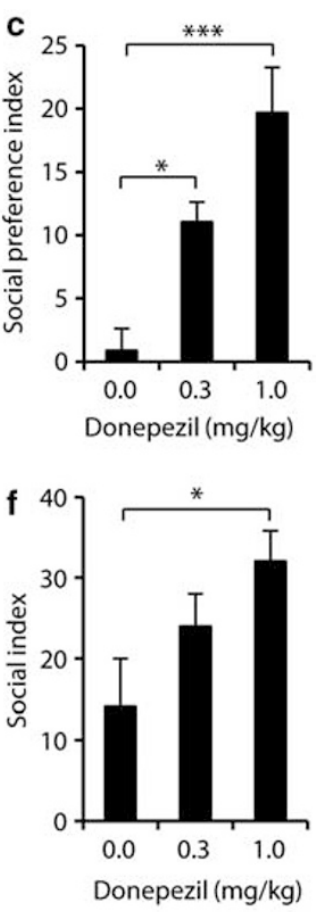

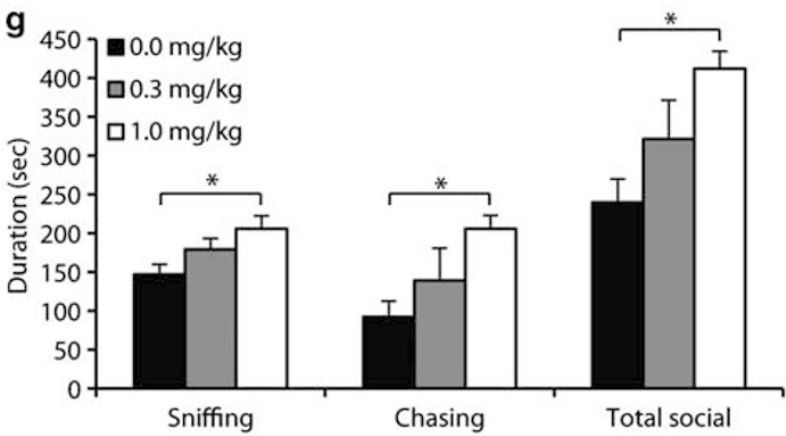

Figure 2 Intraperitoneal (i.p.) administration of Donepezil improved sociability in a dose-dependent manner. BTBR mice were injected i.p. with Donepezil $(0.3$ or $1.0 \mathrm{mg} / \mathrm{kg}$ ) or saline $(0.0 \mathrm{mg} / \mathrm{kg}$ ) for 7 days before testing and $30 \mathrm{~min}$ before each testing day. (a) Scheme of the three-chamber social preference test. One cage contained a stranger mouse (stranger cage) and the other was left empty (empty cage). (b) Sniffing durations of each wire cage. (c) Social preference index, calculated as (time with stranger)/(time with stranger + time with empty cage) $\times 100-50$. (d) Scheme of the 'social' running/jammed wheel assay. The preference of interaction with a stranger over an object was measured by comparing interaction time with a stranger mouse to interaction time with the previously-rewarding jammed-wheel (e). Social preference index ( $f$ ) was calculated as (time with stranger)/(time with stranger + time with jammed wheel) $\times 100-50$. (g) The male-male interaction test. Cumulative durations of sniffing and chasing the intruder mouse, as well as total social interactions, within a I5-min period. Data are presented as mean \pm SEM. ${ }^{*} p=0.09,{ }^{*} p<0.05, * * * 0.0$ I, ${ }^{*} * * * 0.00$ I, n.s: not significant. $N=8 /$ dose.

a

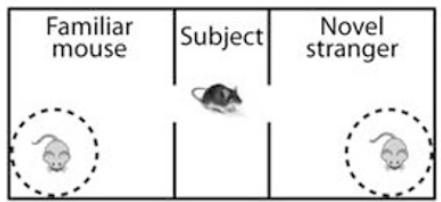

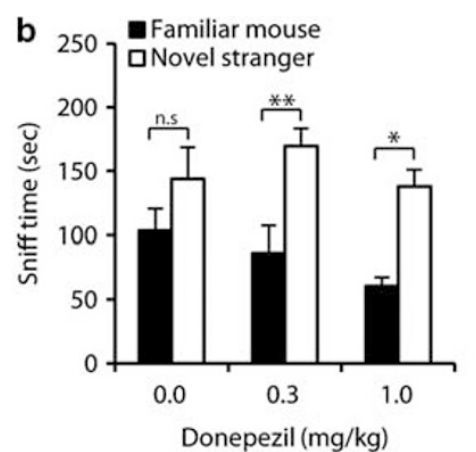

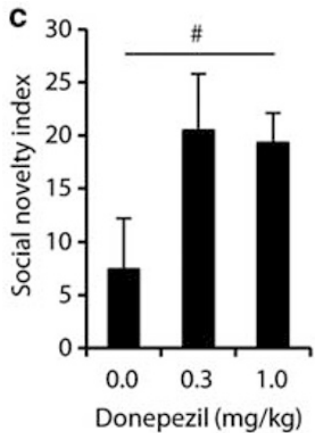

Figure 3 Intraperitoneal (i.p.) administration of Donepezil tended to improve social memory (preference for social novelty). BTBR mice were injected i.p. with Donepezil $(0.3$ or $1.0 \mathrm{mg} / \mathrm{kg}$ ) or saline $(0.0 \mathrm{mg} / \mathrm{kg}$ ) for 7 days before testing and $30 \mathrm{~min}$ before each testing day. (a) Scheme of the three-chamber social novelty preference test. An unfamiliar stranger mouse (novel stranger) was put in the previously empty wire-cage. (b) Sniffing durations of the wire cage containing the novel stranger or familiar mouse. (c) Social novelty preference index, calculated as (time with novel stranger)/(time with novel stranger + time with familiar mouse) $\times 100-50$. Data are presented as mean \pm SEM. ${ }^{\#} p=0.12,{ }^{*} p<0.05$, ${ }^{*} * p<0.0$ I, n.s: not significant. $N=8 /$ dose. 

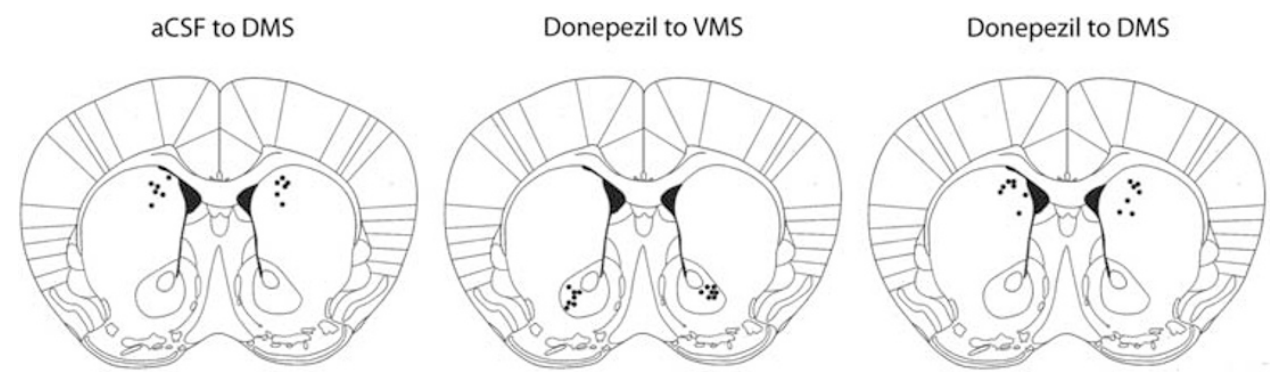

Figure 4 Diagrams illustrating the placement of the tips of the cannulae implanted to BTBR mice in experiment 2. For all mice, anterior-posterior placement was $1.00 \pm 0.05 \mathrm{~mm}$ anterior to bregma. Illustration modified from the coronal section $0.98 \mathrm{~mm}$ anterior to bregma in mouse brain atlas of Franklin and Paxinos (1997). aCSF, artificial cerebrospinal fluid; DMS, dorsomedial striatum; VMS, ventromedial striatum.

mice treated with $0.3 \mathrm{mg} / \mathrm{kg}$ showed a trend toward social preference $(p=0.094)$, and mice treated with $1.0 \mathrm{mg} / \mathrm{kg}$ Donepezil showed a significant preference of the stranger mouse $(p<0.001)$. The dose-dependent improvement was evident also in analysis of the social index (Figure 2c, $\left.\mathrm{F}_{2,21}=12.5, \quad p<0.001\right)$, as the $1.0 \mathrm{mg} / \mathrm{kg}$ group reached significantly $(p<0.001)$ higher scores than the control group, and the $0.3 \mathrm{mg} / \mathrm{kg}$ improved to a lesser extent, yet significantly $(p<0.05)$.

Systemic Donepezil administration also significantly enhanced preference for social interaction in the 'social' running wheel assay (Figure 2d). A significant correlation between stimulus (stranger mouse or jammed-wheel) and dosage was found (Figure 2e, $\mathrm{F}_{2,21}=3.5, p<0.05$ ), as control mice did not significantly prefer the stranger mouse $(p>0.35)$, mice treated with $0.3 \mathrm{mg} / \mathrm{kg}$ showed a significant preference $(p<0.01)$, and the highest preference was found in mice treated with the highest dose $(p<0.001)$. A dosedependent improvement was also found in analysis of the social preference index (Figure $2 \mathrm{f}, \mathrm{F}_{2,21}=5.3, p<0.05$ ), when only mice treated with $1.0 \mathrm{mg} / \mathrm{kg}$ Donepezil exhibited significantly higher scores than the control mice.

In the male-male social interaction test (Figure $2 \mathrm{~g}$ ), $1.0 \mathrm{mg} / \mathrm{kg}$ Donepezil-treated mice spent significantly $(p<0.05)$ more time in sniffing and chasing, as well as total social interaction, compared with saline-treated mice. The difference between the $0.3 \mathrm{mg} / \mathrm{kg}$ group and both other groups was not found significant in any measurement. To note, all mice spent negligible amounts of time $(<1 \mathrm{~s}$ on average) in mounting and aggressive behaviors.

Finally, an improvement was found in the Donepeziltreated groups in the preference for novel social stimulus, as measured in the three-chamber test (Figure 3a). While saline-treated mice did not significantly prefer the novel stranger (Figure $3 b, p>0.4$ ), both doses of the drug resulted in a significant preference for social novelty (Figure $3 b$ ). Furthermore, a trend toward improvement in the social novelty preference index was found (Figure $3 c, F_{2,21}=2.3$, $p=0.12$ ). To note, measurement of total time in chamber yielded a significant preference to the chamber containing the novel stranger for all treatment groups $\left(\mathrm{F}_{1,21}=126.01\right.$, $p<0.001)$, with no interaction between treatment group and time in chamber $\left(\mathrm{F}_{2,21}=1.1, p=0.36\right)$.

No difference between sub-chronic and acute treatments. As shown in Supplementary Table S1, no behavioral differences were found between BTBR mice treated with
$1.0 \mathrm{mg} / \mathrm{kg}$ Donepezil in a sub-chronic regimen compared with an acute treatment with a similar dose.

\section{Experiment 2}

Donepezil administration into the DMS, but not VMS, rescued cognitive rigidity and social deficiency. We next investigated whether intracranial microinjection of Donepezil into the DMS can improve the above described autism-related behaviors. We compared the behavioral performance in the running/jammed wheel assays of a mouse group that received Donepezil into the DMS with two control mouse groups: (a) mouse group that received aCSF into the DMS and (b) mouse group that received Donepezil into the VMS (Figure 4 and Supplementary Figure S2).

Mice from each group acquired the running habit as demonstrated by shorter latencies to start running on the wheel in days 2-4 compared with the first day (Figure 5a, $\mathrm{F}_{3,51}=141.2, p<0.001$ for each group), with no differences between groups $(p>0.8)$. Injection of Donepezil into the DMS, however, resulted in a significant improvement in adjustment to change, as reflected from a significantly lower Jam1/Run4 interaction time ratio (Figure $5 b, F_{2,17}=10.8$, $p<0.001)$ compared with each control group $(p<0.01$ in post hoc analysis).

Social preference was also significantly improved by injection of Donepezil into the DMS, but not into the VMS or aCSF into the DMS. While mice injected with aCSF into the DMS and Donepezil into the VMS spent similar amounts of time in interaction with the stranger mouse or the jammed-wheel (Figure 5c, $p>0.9$ ), mice that received Donepezil into the DMS spent significantly more time with the stranger over interacting with the jammed-wheel $(p<0.01)$. The behavioral difference between groups was reflected in a significant interaction between treatment and stimulus (jammed-wheel or stranger mouse, $F_{2,17}=9.6$, $p<0.01$ ). Social preference index of the mouse group that received Donepezil injection into the DMS was significantly higher than both control groups as well (Figure 5d). The effect of treatment was found significant $\left(F_{1,17}=9.6\right.$, $p<0.01$ ), as the Donepezil to the DMS group presented significantly higher scores than both control groups (Figure $5 \mathrm{~d}, p<0.01$ in post-hoc analysis).

No effect on repetitive behaviors. No significant differences were found in repetitive self-grooming and beddingdigging durations, whether mice were treated systemically 

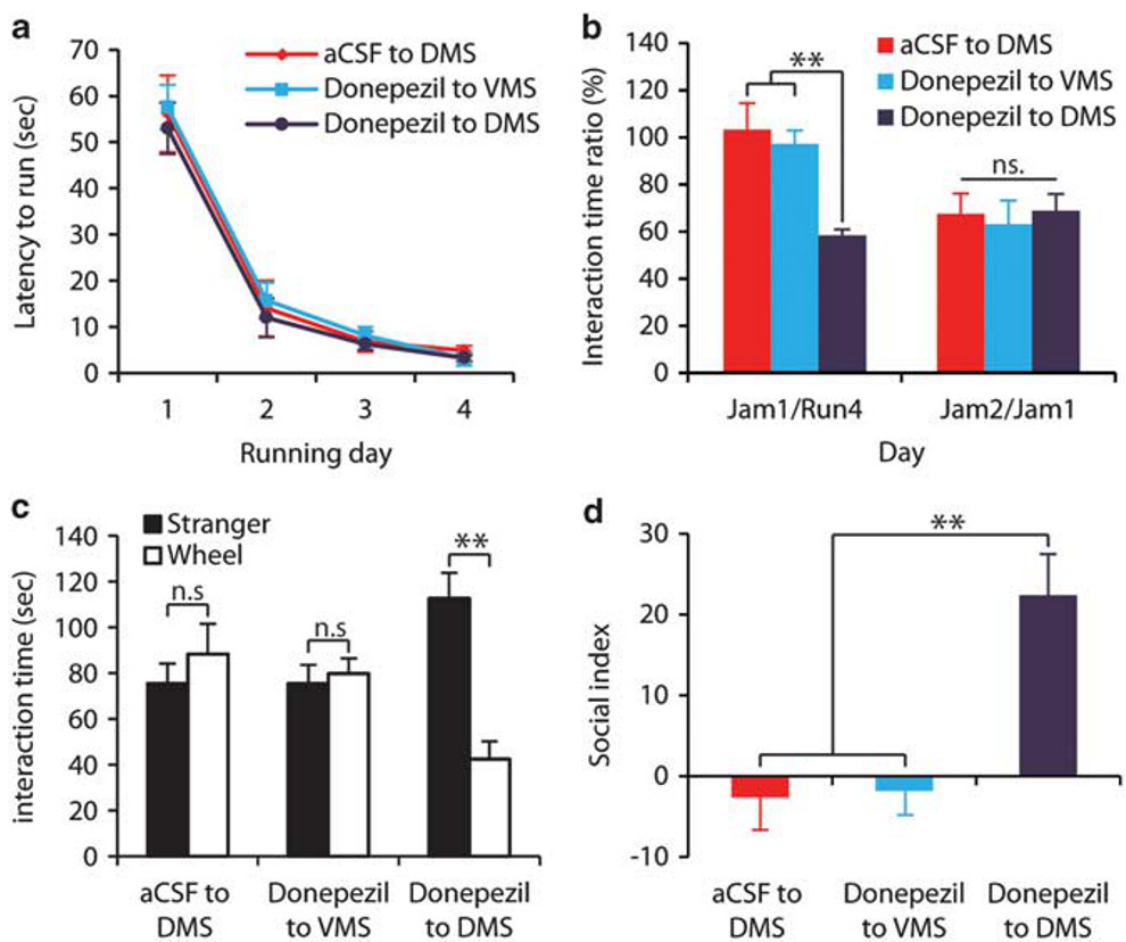

Figure 5 Direct Donepezil administration into the dorso-medial-striatum (DMS), but not into the ventromedial striatum (VMS), rescued cognitive rigidity and social deficiency. Mice were injected with artificial cerebrospinal fluid (aCSF) in the third running day for acclimation to procedure and reduction of stress. In each day from the fourth running day until the end of experiment, mice were injected with either aCSF or Donepezil (according to experimental group). (a) Latency to start running on the wheel in each of the running days, as a measure of habit acquisition. (b) Adjustment to change (ratio between interaction times with the wheel in days Jam I and Run4, left) and memory of the change (ratio between interaction times with the wheel in days Jam2 and Jam I, right). (c) Social interaction: duration of interaction with an unfamiliar mouse (stranger) or with the jammed-wheel (wheel). (d) Social preference index, calculated as (time with stranger)/(time with stranger + time with wheel) $\times 100-50$. DMS, dorsomedial striatum; VMS, ventromedial striatum; aCSF $=0.5$ $\mu \mathrm{l}$ artificial cerebrospinal fluid, Donepezil $=100 \mathrm{ng}$ Donepezil dissolved in $0.5 \mu \mathrm{l}$ aCSF. Data are presented as mean \pm SEM. $* * p<0.01$, n.s $=$ not significant. aCSF to DMS: $N=6$. Donepezil to VMS: $N=7$. Donepezil to DMS: $N=7$.

(Supplementary Figure S3a and b) or intracranially (Supplementary Figure S3c and d) ( $p>0.4$ for all measures).

Control tests. Donepezil-treated mice had slightly higher running duration (Supplementary Figure S4) in comparison with the control group mice. This difference may indicate an improvement in cognitive flexibility reflected in weighing the possible stimuli in the environment and investing more time with the rewarding stimulus, as long as it is rewarding. Alternatively, the difference may be the result of changed locomotion or anxiety levels. Hence, to better define whether Donepezil treatment had effect on locomotion and anxiety-related behaviors, we performed the openfield test on a second cohort of control and drug-treated BTBR mice. No differences between treatment groups were found in general activity (distance moved) (Supplementary Figure S5a, $p>0.7$ ) or anxiety-related behaviors (time spent in the central compartment of the open-field) (Supplementary Figure S5b, $p>0.9$ ).

We also tested the effects of the drug treatment on the behavior of a second mouse strain-the FVB. This stain is considered neurotypical and used as a control mouse model to BTBR (Bolivar et al, 2007; Ellegood et al, 2013a; Moy et al, 2007). FVB mice were tested in the running/jammed wheel test using the exact procedure in which BTBR mice were tested, both systemically and intracranially. Following systemic administration, FVB mice from all treatment groups ran on the wheel similar amounts of time (Supplementary Figure S6a, $p>0.4$ ) and exhibited similar adjustment to change (Supplementary Figure S6b, $p>0.4$ )

Furthermore, similar to systematic drug administrations, intracranially microinjected FVB mice (Supplementary Figure S6c) from all treatment groups ran on the wheel similar amounts of time (Supplementary Figure S6d, $p>0.7)$ and exhibited similar adjustment to change (Supplementary Figure S6e, $p>0.3$ ). Lastly, no difference was found between the control and drug-treated FVB mice in the open-field test $(p>0.7)$. Taken together, treatment with the tested doses of Donepezil did not appear to affect locomotion or anxiety-related behaviors in BTBR mice nor did it affect the neurotypical behaviors of the FVB control strain.

\section{DISCUSSION}

The major finding of this study is that elevation of AChlevels led to a significant improvement in cognitive rigidity, social preference and social interactions, all core symptoms in ASD patients. Systemic treatment with the AChEI Donepezil rescued the autistic-like phenotype in independent and complementary behavioral tests conducted on a well-described mouse model of autism. Similar effects were achieved by direct microinjection into the dorsomedial striatum but not into the ventomedial-striatum. 
The effect of the AChEI Donepezil on cognitive functions was previously examined extensively on neurotypical mice and rats or animal models of Alzheimer disease (Yoo et al, 2007), but, to our knowledge, never on ASD animal models. For example, the positive behavioral effect of the drug was shown with respect to attention, learning, and memory (reviewed in (Yoo et al, 2007)). In addition, Donepezil treatment was demonstrated to increase spontaneous alternations (Spowart-Manning and van der Staay, 2004), improve reversal learning in spatial-tasks (Dong et al, 2005), and relieve social memory deficiency (Riedel et al, 2009).

These reports fit well with our findings. Systemic administration of the drug in the BTBR mouse model of autism improved cognitive flexibility, comparable to the results obtained in spatial reversal learning tests after administration of Donepezil in doses similar to the current study (Yoo et al, 2007). In addition, our results in the social novelty test of untreated BTBR mice agrees with a previous report (Moy et al, 2007), in which these mice spent significantly more time in the chamber containing the novel stranger, but not in physical contact with it. Similarly to (Riedel et al, 2009), treatment with Donepezil improved social memory. To the best of our knowledge, the current study is the first to demonstrate the positive effect of the drug on different aspects of sociability including social preference and malemale social interactions, in a mouse model of autism.

The results obtained from direct infusion of the AChEI Donepezil into the DMS agree with previous studies as well. The group of Ragozzino showed in a series of experiments that disruption of $\mathrm{ACh}$ function in this area results in inability of behavioral shift (McCool et al, 2008; Ragozzino and Choi, 2004). Here, we showed that increment of available ACh in the DMS rescued the cognitively rigid behaviors. Surprisingly, the possible influence of attention and cognitive shift attributed to the cholinergic system and the striatum on social behavior was never thoroughly tested. Nevertheless, a recent study in human reported on loss of empathy and emotion recognition following focal caudate nucleus lesion (Kemp et al, 2013). We found that Donepezil microinjection into the DMS, but not VMS, positively affected social interaction and preference. These therapeutic effects may be explained by reduced cognitive rigidity, resulting in increased social attention, which elevated sociability levels (Avale et al, 2011).

Interestingly, two independent groups (Klin et al, 2007; Turner-Brown et al, 2011) found a correlation between the amount of time autistics invest in circumscribed interests and interference with social interactions. We argue, based on the well-established positive effect of AChEIs on cognition and improved sociability reported here, that Donepezil can enhance cognitive flexibility, thus lead to elevated social attention and improved sociability. Hence, we suggest that future clinical trials with AChEIs will assess the correlation between circumscribed interests and social interference in ASD patients. Clinical studies of this correlation may also close the gap between the clear-cut results of the current study and the only tenuous clinical effects (Handen et al, 2011).

Our findings also add the imperative domain of motor repetitive behaviors that was not tested preclinically with Donepezil before. The drug failed to relieve repetitive self-grooming and digging, similarly to a lack of clinical influence of the drug on the stereotypes subscale of ASD (Hardan and Handen, 2002). A plausible explanation to these findings is that initiation and cessation of complex motor stereotypies depend on the balance between dopamine and AChRs in the striatum (Gao and Singer, 2013), thus augmentation of total cholinergic levels resulted in a mixed behavioral outcome.

In the neuronal level, thalamo-cortical cholinergic projections have been shown to modulate the release of the excitatory neurotransmitter glutamate (Mesulam et al, 1983). On the other hand, striatal cholinergic interneurons change their firing-pattern in response to environmental changes, and modulate the activity of the spiny projections neurons (Goldberg and Reynolds, 2011). The projection neurons, in turn, are the source of the $\gamma$-aminobutyric acid (GABA)ergic inhibitory outcome of the striatum (Graybiel et al, 1994). Within the cortex, ACh has distinct excitatory and inhibitory effects on different layers, depending on receptor-type distribution (Eggermann and Feldmeyer, 2009). Additionally, because ACh regulates CNS development, including growth, differentiation, and plasticity, it has an impact on the representational differentiation of excitatory and inhibitory synapses (Lauder and Schambra, 1999). Therefore, ACh has an important role in modulating the balance between excitation and inhibition (E/I) in the brain. Autism has been hypothesized to arise from disrupted $\mathrm{E} / \mathrm{I}$ balance within neuronal microcircuitry (Rubenstein and Merzenich, 2003), and recently it was demonstrated that increased inhibition moderately ameliorated the social behavior deficits in mice subjected to elevation of cellular E/I balance (Yizhar et al, 2011).

The BTBR strain is a promising model to study the importance of striatal ACh modulation of the E/I balance and its effect on autism. Recent studies demonstrated that low levels of ACh (McTighe et al, 2013) and reduction in striatal size (Ellegood et al, 2013b) accompany the aberrant behavior of this strain. Additionally, recent indirect evidence suggests E/I imbalance involvement in BTBR symptomology, as pharmacological modulation of glutamatergic synapses rescues these behaviors (Silverman et al, 2012). Hence, our findings suggest of interplay between $\mathrm{ACh}$ imbalance and endophenotypes of ASD associated with social deficiencies and cognitive flexibility. Future investigation of the modulatory effect of ACh on the E/I balance in autism-related mouse models should be performed to further elucidate the role of ACh action in the CNS in ASD symptoms, specifically related to attention, flexibility, and their association with social preference. This may potentially open a new venue for therapeutic interventions in ASD patients.

\section{FUNDING AND DISCLOSURE}

This work was supported by the Nella and Leon Benoziyo Center, Kekst Family Institute for Medical Genetics, and Jonathan and Joan Birnbach foundation (TK). The authors declare no conflict of interest.

\section{ACKNOWLEDGEMENTS}

We thank M Dayan, L Edry, Y Sofer, N Barak, and L Khalfin for their assistance in conducting the experiments. We also 
thank A Weissbrod for his assistance with designing and building the experimental set-up.

\section{REFERENCES}

Arnold H, Burk J, Hodgson E, Sarter M, Bruno J (2002). Differential cortical acetylcholine release in rats performing a sustained attention task versus behavioral control tasks that do not explicitly tax attention. Neuroscience 114: 451-460.

Association AP, DSM-IV (2000): APATF on (American Psychiatric Pub). Diagnostic and statistical manual of mental disorders: DSM-IV-TR.

Avale ME, Chabout J, Pons S, Serreau P, Chaumont FD, OlivoMarin J-C et al (2011). Prefrontal nicotinic receptors control novel social interaction between mice. FASEB J 25: 2145-2155.

Bolivar VJ, Walters SR, Phoenix JL (2007). Assessing autism-like behavior in mice: variations in social interactions among inbred strains. Behav Brain Res 176: 21-26.

Buckley AW, Sassower K, Rodriguez AJ, Jennison K, Wingert K, Buckley J et al (2011). An open label trial of Donepezil for enhancement of rapid eye movement sleep in young children with autism spectrum disorders. J Child Adolescent Psychopharmacol 21: 353-357.

Canitano R, Scandurra V (2011). Psychopharmacology in autism: an update. Progress Neuro-Psychopharmacol Biol Psychiatry 35: $18-28$.

Chadman KK, Guariglia SR (2012). The BTBR T+tf/J (BTBR) mouse model of autism. Autism S1: 009.

Chez M, Buchanan T, Becker M, Kessler J, Aimonovitch M, Mrazek S (2003). Donepezil hydrochloride: a double-blind study in autistic children. J Pediatric Neurol 1: 83-88.

Dam D, Van, Abramowski D, Staufenbiel M, De Deyn PP (2005). Symptomatic effect of donepezil, rivastigmine, galantamine and memantine on cognitive deficits in the APP23 model. Psychopharmacol (Berl) 180: 177-190.

Deutsch SI, Urbano MR, Neumann SA, Burket JA, Katz E (2010). Cholinergic abnormalities in autism. Clinical Neuropharmacol 33: $114-120$

Dong H, Csernansky CA, Martin MV, Bertchume A, Vallera D, Csernansky JG (2005). Acetylcholinesterase inhibitors ameliorate behavioral deficits in the Tg2576 mouse model of Alzheimer's disease. Psychopharmacol (Berl) 181: 145-152.

Eggermann E, Feldmeyer D (2009). Cholinergic filtering in the recurrent excitatory microcircuit of cortical layer 4. PNAS 106: $11753-11758$.

Ellegood J, Babineau BA, Henkelman RM, Lerch JP, Crawley JN (2013a). Neuroanatomical analysis of the BTBR mouse model of autism using magnetic resonance imaging and diffusion tensor imaging. NeuroImage 70: 288-300.

Ellegood J, Babineau BA, Henkelman RM, Lerch JP, Crawley JN (2013b). Neuroanatomical analysis of the BTBR mouse model of autism using magnetic resonance imaging and diffusion tensor imaging. NeuroImage 70: 288-300.

Franklin KBJ, Paxinos G (1997). The mouse brain atlas in stereotaxic coordinates. Academic Press: San Diego, CA, USA.

Friedman SDSD (2006). GRay and white matter brain chemistry in young children with autism. Arch Gen Psychiatry 63: 786-794.

Gao S, Singer HS (2013). Complex motor stereotypies: an evolving neurobiological concept. Future Neurology 8: 273-285.

Goldberg JA, Reynolds JNJ (2011). Spontaneous firing and evoked pauses in the tonically active cholinergic interneurons of the striatum. Neuroscience 198: 27-43.

Grahn JA, Parkinson JA, Owen AM (2008). The cognitive functions of the caudate nucleus. Progress Neurobiol 86: 141-155.

Granon S, Faure P, Changeux J-P (2003). Executive and social behaviors under nicotinic receptor regulation. PNAS 100: $9596-9601$.
Graybiel AM, Aosaki T, Flaherty AW, Kimura M (1994). The basal ganglia and adaptive motor control. Science 265: 1826-1831.

Guariglia SR, Jenkins EC Jr., Chadman KK, Wen GY (2011). Chlorination byproducts induce gender specific autistic-like behaviors in CD-1 mice. NeuroToxicol 32: 545-553.

Hallmayer J, Cleveland S, Torres A, Phillips J, Cohen B, Torigoe T et al (2011). Genetic heritability and shared environmental factors among twin pairs with autism. Arch Gen Psychiatry 68: 1095-1102.

Handen BL, Johnson CR, McAuliffe-Bellin S, Murray PJ, Hardan AY (2011). Safety and efficacy of Donepezil in children and adolescents with autism: neuropsychological measures. J Child Adolescent Psychopharmacol 21: 43-50.

Hardan AY, Handen BL (2002). A retrospective open trial of adjunctive donepezil in children and adolescents with autistic disorder. J Child Adolesc Psychopharmacol 12: 237-241.

Karvat G, Kimchi T (2012). Systematic autistic-like behavioral phenotyping of 4 mouse strains using a novel wheel-running assay. Behav Brain Res 233: 405-414.

Kemp J, Berthel M-C, Dufour A, Després O, Henry A, Namer IJ et al (2013). Caudate nucleus and social cognition: neuropsychological and SPECT evidence from a patient with focal caudate lesion. Cortex 49: 559-571.

Kemper TL, Bauman M (1998). Neuropathology of infantile Autism. J Neuropathol Experimental Neurol 57: 645-652.

Klin A, Danovitch JH, Merz AB, Volkmar FR (2007). Circumscribed interests in higher functioning individuals with autism spectrum disorders: an exploratory study. Res Practice Persons with Severe Disabilities 32: 89-100.

Lauder JM, Schambra UB (1999). Morphogenetic roles of acetylcholine. Environ Health Perspect 107: 65-69.

Leblond CS, Heinrich J, Delorme R, Proepper C, Betancur C, Huguet $\mathrm{G}$ et al (2012). Genetic and functional analyses of SHANK2 mutations suggest a multiple hit model of autism Spectrum disorders. PLoS Genet 8: e1002521.

McConville BJ, Sanberg PR, Fogelson MH, King J, Cirino P, Parker $\mathrm{KW}$ et al (1992). The effects of nicotine plus haloperidol compared to nicotine only and placebo nicotine only in reducing tic severity and frequency in Tourette's disorder. Biol Psychiatry 31: 832-840.

McCool MF, Patel S, Talati R, Ragozzino ME (2008). Differential involvement of M1-type and M4-type muscarinic cholinergic receptors in the dorsomedial striatum in task switching. Neurobiol Learn Mem 89: 114-124.

McTighe SM, Neal SJ, Lin Q, Hughes ZA, Smith DG (2013). The BTBR mouse model of autism spectrum disorders has learning and attentional impairments and alterations in acetylcholine and kynurenic acid in prefrontal cortex. PLoS One 8: e62189.

Mesulam M-M, Mufson EJ, Wainer BH, Levey AI (1983). Central cholinergic pathways in the rat: An overview based on an alternative nomenclature (Ch1-Ch6). Neuroscience 10: 1185-1201.

Mikhail FM, Lose EJ, Robin NH, Descartes MD, Rutledge KD, Rutledge SL et al (2011). Clinically relevant single gene or intragenic deletions encompassing critical neurodevelopmental genes in patients with developmental delay, mental retardation, and/or autism spectrum disorders. AmJ Medical Genet Part A 155: 2386-2396.

Moy SS, Nadler JJ, Young NB, Perez A, Holloway LP, Barbaro RP et al (2007). Mouse behavioral tasks relevant to autism: phenotypes of 10 inbred strains. Behav Brain Res 176: 4-20.

Mukaetova-Ladinska EB, Westwood J, Perry EK (2010). Cholinergic component of autism spectrum disorder. The Neurochemical Basis of Autism 129-161.

Pepeu G, Giovannini MG (2004). Changes in acetylcholine extracellular levels during cognitive processes. Learn Mem 11: 21-27.

Ragozzino ME, Choi D (2004). Dynamic changes in acetylcholine output in the medial striatum during place reversal learning. Learn Mem 11: 70-77. 
Ragozzino ME, Pal SN, Unick K, Stefani MR, Gold PE (1998). Modulation of hippocampal acetylcholine release and spontaneous alternation scores by intrahippocampal glucose injections. J Neurosci 18: 1595-1601.

Riedel G, Kang SH, Choi DY, Platt B (2009). Scopolamine-induced deficits in social memory in mice: reversal by donepezil. Behav Brain Res 204: 217-225.

Rubenstein JLR, Merzenich MM (2003). Model of autism: increased ratio of excitation/inhibition in key neural systems. Genes, Brain Behav 2: 255-267.

Sears LL, Vest C, Mohamed S, Bailey J, Ranson BJ, Piven J (1999). An MRI study of the basal ganglia in autism. Prog NeuroPsychopharmacol Biol Psychiatry 23: 613-624.

Silverman JL, Smith DG, Rizzo SJS, Karras MN, Turner SM, Tolu SS et al (2012). Negative allosteric modulation of the mGluR5 receptor reduces repetitive behaviors and rescues social deficits in mouse models of autism. Sci Transl Med 4: 131ra51131 ra51.

Sokol DK, Dunn DW, Edwards-Brown M, Feinberg J (2002). Hydrogen proton magnetic resonance spectroscopy in autism: preliminary evidence of elevated choline/creatine ratio. J Child Neurol 17: 245-249.
Spowart-Manning L, van der Staay F (2004). The T-maze continuous alternation task for assessing the effects of putative cognition enhancers in the mouse. Behav Brain Res 151: 37-46.

Srivastava RK, Agarwal M, Pundhir A (2011). Role of Donepezil in autism: its conduciveness in psychopharmacotherapy. Case Rep Psychiatry 2011: 1-2.

Turner-Brown LM, Lam KSL, Holtzclaw TN, Dichter GS, Bodfish JW (2011). Phenomenology and measurement of circumscribed interests in autism spectrum disorders. Autism 15: 437-456.

Walker BR, Diefenbach KS, Parikh TN (2007). Inhibition within the nucleus tractus solitarius (NTS) ameliorates environmental exploration deficits due to cerebellum lesions in an animal model for autism. Behav Brain Res 176: 109-120.

Wang H, Tang XC (1998). Anticholinesterase effects of huperzine A, E2020, and tacrine in rats. Zhongguo Yao Li Xue Bao 19: 27-30.

Yizhar O, Fenno LE, Prigge M, Schneider F, Davidson TJ, O'Shea DJ et al (2011). Neocortical excitation/inhibition balance in information processing and social dysfunction. Nature 477: 171-178.

Yoo JH, Valdovinos MG, Williams DC (2007). Relevance of Donepezil in enhancing learning and memory in special populations: a review of the literature. J Autism Dev Disord 37: 1883-1901.

Supplementary Information accompanies the paper on the Neuropsychopharmacology website (http://www.nature.com/npp) 